\title{
Clinical significance of growth factor receptor EGFR and angiogenesis regulator VEGF-R2 in patients with ovarian cancer at FIGO stages I-II
}

\author{
INGIRIDUR SKIRNISDOTTIR $^{1}$, HELENA ÅKERUD ${ }^{2}$ and TOMAS SEIDAL ${ }^{3}$ \\ ${ }^{1}$ Department of Women's and Children's Health, ${ }^{2}$ Department of Immunology, Genetics and Pathology, Uppsala University, \\ SE-751 85 Uppsala; ${ }^{3}$ Department of Pathology, Halmstad Medical Center Hospital, SE-301 85 Halmstad, Sweden
}

Received November 15, 2017; Accepted June 7, 2018

DOI: $10.3892 /$ ijo.2018.4511

\begin{abstract}
The aim of the present retrospective cohort study was to investigate the prognostic effect of epidermal growth factor receptor (EGFR) and the angiogenesis regulator vascular endothelial growth factor receptor 2 (VEGF-R2) on disease-free survival (DFS) rate and recurrent disease, and their association with clinicopathological characteristics in 131 patients with International Federation of Gynecology and Obstetrics (FIGO) stages I-II epithelial ovarian cancer. The techniques of tissue microarrays and immunohistochemistry were used for the positive detection of the markers. The frequency of positive staining in tumors for EGFR was $24 \%$ and for VEGF-R2 was 77\%. Across the cohort, there was a total of 34/131 recurrences (26\%) and the 5-year DFS rate was $68 \%$. In a multivariate logistic regression analysis with recurrent disease as the endpoint, FIGO stage (OR=9.7), type (I/II) of tumor $(\mathrm{OR}=3.0)$ and VEGF-R2 status $(\mathrm{OR}=0.2)$ were all found to be independent predictive factors in the cohort of patients $(n=131)$. For patients with non-serous tumors $(n=78)$, the FIGO stage $(\mathrm{OR}=76)$, type $(\mathrm{I} / \mathrm{II})$ of tumor $(\mathrm{OR}=44)$, EGFR status $(\mathrm{OR}=0.05)$ and VEGF-R2 status $(\mathrm{OR}=0.008)$ were all significant and independent predictive factors. On comparing the four subgroups, in terms of concomitant EGFR and VEGF-R2 status, in a survival analysis, the subgroup of patients $(n=21)$ with concomitant positive expression of EGFR and VEGF-R2 had a 5-year DFS rate of $100 \%$. Therefore, the prognostic effect of EGFR and VEGF-R2 for recurrent disease and survival rates was confirmed by the above findings. Certain results in the present study were not in line with results from previous studies on the prognostic effect of EGFR and VEGF-R2. An increasing number of preclinical and clinical observations
\end{abstract}

Correspondence to: $\mathrm{Dr}$ Ingiridur Skirrnisdottir, Department of Women's and Children's Health, Uppsala University, Dag Hammarskjölds väg 20, SE-751 85 Uppsala, Sweden

E-mail: ingiridur.skirnisdottir@kbh.uu.se

Key words: epidermal growth factor receptor, tumor angiogenesis, vascular endothelial growth factor receptor 2 have shown that the process of angiogenesis remains to be fully elucidated. Therefore, one of the challenges for future ovarian cancer investigations is to identify which biomarkers may be used as predictive and prognostic markers.

\section{Introduction}

The epidermal growth factor receptor (EGFR) family consists of four members: EGFR, human epidermal growth factor receptor (HER)2, HER3 and HER4. Structurally, the EGFR family consists of an extracellular ligand-binding domain, a single transmembrane-spanning region, and an intracellular region containing the kinase domain (1). Overexpression of the EGFR protein has been detected in 9-62\% of cases of human ovarian cancer in previous studies, and the differences in frequencies in these studies likely reflect the use of different antibodies and cutoffs for overexpression (2). EGFR gene amplification or protein overexpression occurs across all epithelial ovarian cancer histological subtypes, and increased expression of EGFR has been associated with high tumor grade, a high cell proliferation index and poor patient outcome $(1,3)$. It was shown in a previous study (4) on human ovarian carcinoma cells and the expression of EGFR that EGFR regulates cell adhesion proteins that may enhance cell growth and invasiveness.

For solid tumor growth, tumor angiogenesis is essential, and the passage of carcinoma cells through the basic membrane and the infiltration of adjacent tissues are key stages in the development of ovarian cancer (4). Therefore, angiogenesis is an important process for the creation of blood and lymphatic vessels, which sustain the growth of the tumor (5). It is known, that VEGF-R2 acts as a receptor for VEGF-A during neovascularization (6).

The prognostic value of the overexpression of EGFR has been associated with contradictory results. A poor prognosis was reported in a previous study (3) on a population of 106 patients with International Federation of Gynecology and Obstetrics (FIGO) stage I-II disease, and from a study (7) on 398 patients with FIGO stages I-IV epithelial ovarian cancer. However, among studies that showed no prognostic effect of EGFR status, two included a large number of patients with epithelial ovarian cancer, including 80 patients at FIGO stage III (8) and 93 patients at FIGO stages III-IV (9). The 
results from studies concerning the prognostic value of VEGF-R2 in ovarian cancer have also been associated with contradictory results $(5,6)$.

In a previous study (10), VEGF-R2 status was significantly $(\mathrm{P}=0.011)$ associated with type II tumors. Furthermore, recurrent disease occurred more frequently $(\mathrm{P}=0.049)$ in a subgroup of patients with VEGF-R2-negative tumors. In a survival analysis, patients from the subgroup with VEGF-R2-positive tumors had a significantly higher 5-year disease-free survival (DFS) rate of $90 \%$, compared to $66 \%$ in the subgroup of patients with VEGF-R2-negative tumors. The objective of the present study was to investigate the prognostic value of the growth factor receptor EGFR and the angiogenesis regulator VEGF-R2, and examine their association with clinicopathological factors, recurrent disease and DFS rates in 131 patients with epithelial ovarian cancer at FIGO stages I-II.

\section{Materials and methods}

Studypopulation.In the Uppsala-Örebro Medical Region during the 5-year period between 1st January 2000 and 31st December 2004, a total of 140 consecutive patients with FIGO stage I-II epithelial ovarian cancer, who underwent primary surgery and post-surgical chemotherapy, were recruited to the present study. All tissue samples were collected with the patients' informed consent and were in compliance with the Declaration of Helsinki (11), and were used in accordance with the Swedish Biobank Legislation and Ethical Review Act approved by the Uppsala Ethical Review Board (Uppsala, Sweden; decision ref. UPS-03-477). Of the 140 patients, a total of 131 patients who agreed to participate in the study were included. There were 131 available tumors for the analysis of EGFR and there were 130 available tumors for the analysis of VEGF-R2.

The primary surgery was performed at nine surgical gynecological departments. The staging procedure was performed at the time of primary surgery. According to the European Organization for the Research and Treatment of Cancer surgical staging (12), modified surgical staging was undertaken in 34 (26\%) of the 131 cases and, according to the same guidelines, surgical staging was regarded as minimal or inadequate in the remaining 77 (74\%) patients.

The characteristics of the patients are summarized in Table I, including the age, body mass index (BMI), performance status of the patients (World Health Organization), FIGO stage, serous/non-serous histology and type of ovarian tumor (type I and type II). All patients had post-surgical chemotherapy 4-6 weeks following primary surgery, most commonly with paclitaxel $\left(175 \mathrm{mg} / \mathrm{m}^{2}\right)$ and carboplatin $(\mathrm{AUC}=5)$ at 3 -week intervals, usually for four courses $(n=105)$, or single-drug carboplatin for four to six courses $(n=26)$. The mean follow-up time was 65 months (range 5-110 months). The definition of survival was taken as the date of confirmed histological diagnosis following primary surgery to the date of recurrence, the patient succumbing to mortality, or their final visit.

Sampling and tissue microarray construction of ovarian cancer tissue. Paraffin-embedded tumor tissue from primary surgery was used. Following staining with hematoxylin and eosin, the tumors were classified and graded by a single pathologist. The tissue microarrays were constructed as described
Table I. Patient characteristics..

\begin{tabular}{|c|c|}
\hline Characteristic & $\mathrm{n}(\%)$ \\
\hline Median age (years) & 59.0 (range 25-84) \\
\hline \multicolumn{2}{|l|}{ BMI } \\
\hline $\mathrm{BMI} \leq 25$ & $69(53.9)$ \\
\hline $\mathrm{BMI}>25$ & $59(46.1)$ \\
\hline \multicolumn{2}{|l|}{ WHO performance status } \\
\hline 0 & $37(28.2)$ \\
\hline 1 & $66(50.4)$ \\
\hline 2 & $21(16.0)$ \\
\hline 3 & $6(4.6)$ \\
\hline \multicolumn{2}{|l|}{ FIGO stage } \\
\hline IA & $39(29.7)$ \\
\hline IB & $6(4.6)$ \\
\hline IC & $66(50.4)$ \\
\hline II & $20(15.3)$ \\
\hline \multicolumn{2}{|l|}{ Histopathology $\mathrm{y}^{\mathrm{a}}$} \\
\hline Serous ovarian tumors & $51(39.2)$ \\
\hline Non-serous ovarian tumors & $78(60.8)$ \\
\hline Mucinous & $20(25.6)$ \\
\hline Endometrioid & $42(53.8)$ \\
\hline Clear cell & $16(20.5)$ \\
\hline \multicolumn{2}{|l|}{ Types of ovarian tumors ${ }^{\mathrm{b}}$} \\
\hline Type I tumors & $79(65.8)$ \\
\hline Low-grade (G1) serous & 14 \\
\hline Mucinous $(\mathrm{G} 1+\mathrm{G} 2+\mathrm{G} 3)$ & 20 \\
\hline Low-grade endometrioid (G1+G2) & 29 \\
\hline Clear cell & 16 \\
\hline Type II tumors & $52(34.2)$ \\
\hline High-grade $(\mathrm{G} 2+\mathrm{G} 3)$ serous & 37 \\
\hline High-grade (G3) endometrioid & 13 \\
\hline Anaplastic & 2 \\
\hline
\end{tabular}

${ }^{a}$ Two tumors of anaplastic histology were not included. ${ }^{b}$ Tumors were divided into type I and type II tumors according to the combination of histological subtype and FIGO-grade. With exception of histopathology, all information in this table is adapted from Table II of ref. 10. FIGO, International Federation of Gynecology and Obstetrics; BMI, body mass index.

previously (13). Briefly, tumor tissues were embedded in paraffin and 5- $\mu \mathrm{m}$ sections stained with hematoxylin and eosin were obtained to select representative areas for biopsies. The core tissue biopsy specimens (diameter, $0.6 \mathrm{~mm}$ ) were obtained from these regions of individual donor paraffin blocks and precisely arrayed into a new recipient paraffin block using a custom-built instrument. Two tissue core specimens (diameter, $0.6 \mathrm{~mm}$ ) from all 131 ovarian carcinomas were arranged in three recipient paraffin blocks. A single pathologist (T.S.) verified all hematoxylin and eosin-stained sections and the presence of tumor tissue on the arrayed samples using a Nikon Eclipse Ni microscope (Nikon Corporation, Tokyo, Japan). The tissue microarray construction was performed at the Department of Pathology, University Hospital MAS (Malmö, Sweden). 
Table II. Status of protein expression of EGFR and VEGF-R2 in tumors, vs. clinical and pathological features ( $\mathrm{n}=131)$.

\begin{tabular}{|c|c|c|c|c|c|}
\hline Feature & EGFR, $\mathrm{n}(\%)^{+}$ & EGFR $^{-}$ & VEGF-R2 ${ }^{+}$ & & VEGF-R2- \\
\hline Number & $31(24)$ & $100(77)$ & $100(77)$ & & $30(23)$ \\
\hline Age (mean, years) & 61 & 57 & 59 & & 58 \\
\hline P-value (t-test) & \multicolumn{2}{|c|}{0.140} & \multicolumn{3}{|c|}{0.620} \\
\hline \multicolumn{6}{|l|}{ Histopathology $\mathrm{y}^{\mathrm{a}}$} \\
\hline Serous & $10(32)$ & $41(42)$ & $41(42)$ & & $9(30)$ \\
\hline Non-serous & $21(68)$ & $57(58)$ & $57(58)$ & & $21(70)$ \\
\hline P-value $\left(\chi^{2}\right)$ & \multicolumn{2}{|c|}{0.342} & \multicolumn{3}{|c|}{0.245} \\
\hline \multicolumn{6}{|l|}{ Tumor grade } \\
\hline $\mathrm{G} 1+\mathrm{G} 2$ & $24(77)$ & $51(51)$ & $56(56)$ & & $19(63)$ \\
\hline G3 & $7(23)$ & $49(49)$ & $44(44)$ & & $11(37)$ \\
\hline P-value $\left(\chi^{2}\right)$ & \multicolumn{2}{|c|}{0.009} & \multicolumn{3}{|c|}{0.476} \\
\hline \multicolumn{6}{|l|}{ Type of tumor } \\
\hline Type I & $23(74)$ & $56(56)$ & $54(54)$ & & $24(80)$ \\
\hline $\mathrm{T}$ ype II & $8(26)$ & $44(44)$ & $46(46)$ & & $6(20)$ \\
\hline P-value $\left(\chi^{2}\right)$ & \multicolumn{2}{|c|}{0.070} & \multicolumn{3}{|c|}{0.011} \\
\hline \multicolumn{6}{|l|}{ FIGO stage } \\
\hline IA-IB & $14(45)$ & $31(31)$ & $34(34)$ & & $11(37)$ \\
\hline IC & $15(48)$ & $51(51)$ & $49(49)$ & & $16(53)$ \\
\hline II & $2(7)$ & $18(18)$ & $17(17)$ & & $3(10)$ \\
\hline P-value $\left(\chi^{2}\right)$ & \multicolumn{2}{|c|}{0.175} & \multicolumn{3}{|c|}{0.647} \\
\hline \multicolumn{6}{|l|}{ Recurrent disease } \\
\hline Without & $26(84)$ & $71(71)$ & $78(78)$ & & $18(60)$ \\
\hline With & $5(16)$ & $29(29)$ & $22(22)$ & & $12(40)$ \\
\hline P-value $\left(\chi^{2}\right)$ & \multicolumn{2}{|c|}{0.153} & \multicolumn{3}{|c|}{0.049} \\
\hline
\end{tabular}

${ }^{a}$ Anaplastic tumors $(\mathrm{n}=2)$ were excluded. All information on VEGF-R2 in this table is adapted from Table II of ref. 10. FIGO, International Federation of Gynecology and Obstetrics; EGFR, epidermal growth factor receptor; VEGFR2, vascular endothelial growth factor receptor 2.

Immunohistochemistry (IHC) and interpretation. From each multi-tissue block, $5-\mu$ m-thick sections were cut and placed on coated slides, and dried overnight at $37^{\circ} \mathrm{C}$. The sections were pre-treated by heat-induced epitope retrieval in target retrieval solution (Dako, Glostrup, Denmark; pH 6.0), or EDTA buffer $(\mathrm{pH} 9.0)$, for $7+7 \mathrm{~min}$ in a microwave oven $\left(99^{\circ} \mathrm{C}\right)$. Blocking with peroxidase was performed for $5 \mathrm{~min}$. The slides were counterstained for 2 min with hematoxylin. The following monoclonal primary antibodies were, used: For EGFR, the monoclonal mouse primary antibody EGFR 113 (dilution 1:40) (Novocastra; Leica Biosystems GmbH, Nussloch, Germany) was used, and for VEGF-R2, the polyclonal mouse antibody Flk-1 (dilution 1:40) was used (Santa Cruz Biotechnology, Inc., Dallas, TX, USA), as described in a previous study (10). Using the REAL Envision detection system (Dako), the immunostainings were performed in an Autostainer automated machine (Dako). The IHC analyses and interpretation were performed at the Department of Pathology, Halmstad Medical Central Hospital (Halmstad, Sweden). The IHC staining was interpreted by I.S. and T.S. No information was available on the specific diagnosis or prognosis of the individual cases at the time of evaluation. Of the 131 tumor samples, staining was successful in 131 tumor samples for EGFR, and in 130 available tumor samples for VEGF-R2. A semi-quantitative analysis (14) was performed and the staining was graded as negative,,+++ , and +++ for EGFR and VEGF-R2, and both of the markers were dichotomized into negative and positive $(+,++$ and +++$)$ cases (15). Positive staining for EGFR was characterized by distinct staining of the cytoplasmic membrane, whereas staining for VEGF-R2 was confined to the cytoplasm and the membrane of the tumor cells.

Statistical analysis. Pearson's $\chi^{2}$ test was used to assess proportional differences in univariate analyses. The survival curves were generated using the Kaplan-Meier technique and differences between these curves were, tested with the log-rank test or $\chi^{2}$ test. The logistic regression model was, used for bivariate and multivariate analyses, with recurrent disease as the endpoint. Furthermore, the univariate and multivariate Cox regression model was, used, with DFS as the endpoint. All tests were two-sided, and $\mathrm{P} \leq 0.05$ was, considered to indicate a statistically significant difference. The STATISTICA 13.2 (StatSoft, Inc., Tulsa, OK, USA) statistical package was, used for analyses.

\section{Results}

Background characteristics. The Patients' characteristics are, presented in Table I. The study population was divided into 
Table III. Status of protein expression in tumors of concomitant EGFR and VEGF-R2, vs. clinical and pathological features $(n=130)$.

\begin{tabular}{|c|c|c|c|c|}
\hline Feature & $\begin{array}{c}\mathrm{EGFR}^{+} / \mathrm{VEGF}-\mathrm{R}^{+}{ }^{+} \\
\mathrm{n}(\%)\end{array}$ & $\begin{array}{c}\mathrm{EGFR}^{+} / \mathrm{VEGF}-\mathrm{R}^{-} \\
\mathrm{n}(\%)\end{array}$ & $\begin{array}{c}\mathrm{EGFR}^{-/ V E G F-R} 2^{-} \\
\mathrm{n}(\%)\end{array}$ & $\begin{array}{c}\text { EGFR-/VEGF-R2 } \\
\mathrm{n}(\%)\end{array}$ \\
\hline Number & $21(21)$ & $10(7)$ & $79(61)$ & $20(15)$ \\
\hline \multicolumn{5}{|c|}{ Histopathology ${ }^{a}$} \\
\hline Serous & $6(29)$ & $4(40)$ & $35(45)$ & $5(25)$ \\
\hline Non-serous & $15(71)$ & $6(60)$ & $42(55)$ & $15(75)$ \\
\hline P-value $\left(\chi^{2}\right)$ & \multicolumn{4}{|c|}{0.266} \\
\hline \multicolumn{5}{|l|}{ Tumor grade } \\
\hline $\mathrm{G} 1+\mathrm{G} 2$ & $15(71)$ & $9(90)$ & $40(51)$ & $10(50)$ \\
\hline G3 & $6(29)$ & $1(10)$ & 39 (49) & $10(50)$ \\
\hline P-value $\left(\chi^{2}\right)$ & \multicolumn{4}{|c|}{0.047} \\
\hline \multicolumn{5}{|c|}{ Type of tumors } \\
\hline Type I & $15(71)$ & $8(80)$ & $39(49)$ & $16(80)$ \\
\hline Type II & $6(29)$ & $2(20)$ & $40(51)$ & $4(20)$ \\
\hline P-value $\left(\chi^{2}\right)$ & \multicolumn{4}{|c|}{0.019} \\
\hline \multicolumn{5}{|l|}{ FIGO stage } \\
\hline IA-IB & $10(48)$ & $4(40)$ & $24(30)$ & $7(35)$ \\
\hline $\mathrm{IC}$ & $9(43)$ & $6(60)$ & $40(51)$ & $10(50)$ \\
\hline II & $2(9)$ & $0(00)$ & 15 (19) & $3(15)$ \\
\hline P-value $\left(\chi^{2}\right)$ & \multicolumn{4}{|c|}{0.413} \\
\hline \multicolumn{5}{|c|}{ Recurrent disease } \\
\hline Without & $19(90)$ & $7(70)$ & $59(75)$ & $11(55)$ \\
\hline With & $2(10)$ & $3(30)$ & $20(25)$ & $9(45)$ \\
\hline P-value $\left(\chi^{2}\right)$ & \multicolumn{4}{|c|}{0.079} \\
\hline
\end{tabular}

${ }^{\mathrm{a} A n a p l a s t i c ~ t u m o r s ~}(\mathrm{n}=2)$ were excluded. FIGO, International Federation of Gynecology and Obstetrics; EGFR, epidermal growth factor receptor; VEGFR2, vascular endothelial growth factor receptor 2.

79 type I tumors $(65.8 \%)$ and 52 type II tumors (34.2\%). The majority of the patients $(84.3 \%)$ had stage I disease and the majority (66\%) of the tumors were classified as type I tumors. A primary cure was, achieved in all 131 patients. The total number of recurrences in the complete cohort was 34/131 (26\%), and 22 of these patients $(67 \%)$ succumbed to the disease. Recurrent disease was significantly associated with FIGO grade $(\mathrm{P}=0.030)$, FIGO sub-stage $(\mathrm{P}=0.0005)$, adequate surgical staging $(\mathrm{P}=0.033)$ and residual disease $(\mathrm{P}=0.001)$. In the entire cohort, the 5-year DFS rate was $68 \%$, the disease-specific survival rate was $76 \%$, and the overall survival rate was $71 \%$. The protein expression status (positive/negative) of the growth factor receptor EGFR and the angiogenesis regulator VEGF-R2 (Table II) was, compared in addition to specific clinical and pathological factors. However, no correlation between the protein expression of EGFR and VEGF-R2 was detected $(\mathrm{P}=0.164)$.

EGFR status. Positive expression of EGFR was identified as distinct staining of the cytoplasmic membrane, and positive expression of EGFR was observed in 31 (24\%) of the 131 available tumors. There were no significant differences in mean age between the groups of patients with EGFR-positive and EGFR-negative tumors (61 years, vs. 57 years; $\mathrm{P}=0.140$ ) across the cohort of patients. The EGFR-status (Table II) was not associated with serous/non-serous tumors, FIGO stage or recurrent disease. By contrast, the EGFR status was associated with tumor grade. The EGFR-positive tumors were predominantly of a lower grade $(\mathrm{G} 1+\mathrm{G} 2)$, compared with the EGFR-negative tumors, which were more frequently of a high grade (G3). All 16 tumors with clear cell histology were classified as high grade (G3) tumors. Furthermore, a trend was observed $(\mathrm{P}=0.070)$, that EGFR-positive tumors more were frequently type I tumors. EGFR status was not significantly associated with BMI (dichotomized; $\mathrm{P}=0.478$ ).

VEGF-R2 status. Positive expression of VEGF-R2 was confined to the membrane and cytoplasm, and positive expression of VEGF-R2 was observed in $100(77 \%)$ of the available 130 tumors, as shown in Table II. Furthermore, the association between VEGF-R2 status in tumors, and clinical and pathological features, was, presented in an earlier study (10).

EGFR VEGF-R2 status. As presented in Table III, the differences in clinical and pathological variables with concomitant EGFR and VEGF-R2 status in four subgroups were limited to tumor grade (G1+G2 / G3) and type (I/II). The EGFR-positive tumors were predominantly of a lower grade $(\mathrm{G} 1+\mathrm{G} 2)$ with/without concomitant VEGF-R2-positive expression. Furthermore, VEGF-R2-positive tumors with concomitant EGFR-positive or EGFR-negative expression 


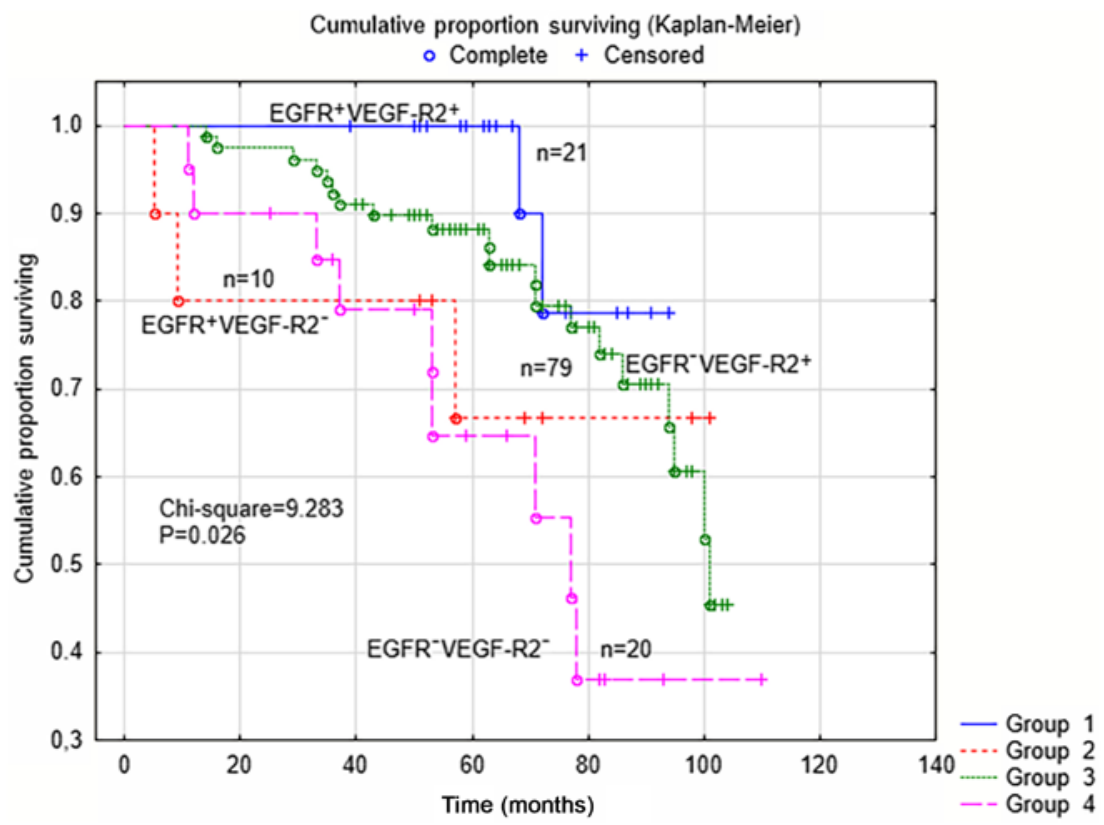

Figure 1. Survival analysis showed that patients $(n=21)$ in the subgroup with concomitant positive expression of EGFR and VEGF-R2 in tumors had a significantly higher 5-year disease-free survival rate compared with patients in the other three subgroups. EGFR, epithelial growth factor receptor; VEGF-R2, vascular endothelial growth factor receptor 2.

were usually type II tumors. There were different outcomes in the four subgroups of patients $(n=130)$ in terms of the EGFR and VEGF-R2 status of the tumors, as shown in Fig. 1. In the survival analysis $\left(\mathrm{P}=0.026 ; \chi^{2}=9.283\right)$, it was shown that the patients $(n=21)$ in the subgroup with concomitant positive expression of EGFR and VEGF-R2 had 5-year DFS rates of $100 \%$. However, no differences between the four subgroups were found according to different surgical staging $(\mathrm{P}=0.640)$ or the type of post-surgical treatment, (paclitaxel and carboplatin, vs. single drug carboplatin; $\mathrm{P}=0.198)$.

Serous tumors. For the serous tumors $(\mathrm{n}=51)$ the EGFR status of the tumors was associated with tumor grade $(\mathrm{P}=0.018)$; 9/10 (90\%) of serous tumors with EGFR-positive expression were of low grade $(\mathrm{G} 1+\mathrm{G} 2)$ compared with 20/41 (49\%) of the tumors with EGFR-negative expression. The VEGF-R2 status of the tumors was not associated with any of the variables shown in Table II, nor with BMI (dichotomized).

Patients who had EGFR-positive tumors of type $I(n=79)$ were older (62 vs. 55 years; $\mathrm{P}=0.039$ ) than the patients with EGFRnegative tumors. For patients with type I tumors (serous low grade), recurrent disease was significantly associated $(\mathrm{P}=0.008)$ with VEGF-R2 negative expression, however, no further differences in clinical or pathological features were detected for the type of tumor, according to VEGF-R2 status, in the present study.

Non-serous tumors. For the non-serous tumors $(n=78)$ the EGFR status of the tumors was associated with age; patients with EGFR-positive tumors were older (62 vs. 55 years; $\mathrm{P}=0.039$ ) than patients with EGFR-negative tumors. The EGFR status of the tumors was not associated with BMI (dichotomized; $\mathrm{P}=0.641$ ).

The EGFR-status of non-serous tumors was associated with recurrent disease $(\mathrm{P}=0.027)$, as shown in Table IV. Only one (5\%) patient had recurrent disease in the subgroup
Table IV. Status of protein expression in tumors of EGFR and VEGF-R2 vs. clinical and pathological features in non-serous tumors $(\mathrm{n}=78)$.

\begin{tabular}{|c|c|c|c|c|}
\hline Feature & $\begin{array}{c}\mathrm{EGFR}^{+} \\
\mathrm{n}(\%)\end{array}$ & $\begin{array}{c}\text { EGFR }^{-} \\
\mathrm{n}(\%)\end{array}$ & $\begin{array}{c}\text { VEGF-R2 } 2^{+} \\
\text {n }(\%)\end{array}$ & $\begin{array}{c}\text { VEGF-R2- } \\
\mathrm{n}(\%)\end{array}$ \\
\hline Number & $21(27)$ & $57(73)$ & $57(73)$ & $21 \quad(27)$ \\
\hline \multicolumn{5}{|l|}{ Histopathology } \\
\hline Mucinous & $4(19)$ & $16(28)$ & $13(23)$ & $7 \quad(33)$ \\
\hline Endometroid & $13(62)$ & $29(51)$ & $34(60)$ & $8 \quad(38)$ \\
\hline Clear cell & $4(19)$ & $12(21)$ & $10(17)$ & $6 \quad(29)$ \\
\hline P-value $\left(\chi^{2}\right)$ & \multicolumn{2}{|c|}{0.649} & \multicolumn{2}{|c|}{0.234} \\
\hline \multicolumn{5}{|l|}{ Tumor grade } \\
\hline $\mathrm{G} 1+\mathrm{G} 2$ & $5(24)$ & $12(21)$ & $13(23)$ & 4 (19) \\
\hline G3 & $16(76)$ & $45(79)$ & $44(77)$ & $17(81)$ \\
\hline P-value $\left(\chi^{2}\right)$ & \multicolumn{2}{|c|}{0.739} & \multicolumn{2}{|c|}{0.721} \\
\hline \multicolumn{5}{|l|}{ Type of tumor } \\
\hline Type I & $19(90)$ & $46(81)$ & $44(77)$ & $21(100)$ \\
\hline Type II & $2(10)$ & $11(19)$ & $13(23)$ & $0 \quad(00)$ \\
\hline P-value $\left(\chi^{2}\right)$ & \multicolumn{2}{|c|}{0.304} & \multicolumn{2}{|c|}{0.016} \\
\hline \multicolumn{5}{|l|}{ FIGO stage } \\
\hline IA-IB & $10(48)$ & $20(35)$ & $21(37)$ & $9 \quad(43)$ \\
\hline IC & $9(43)$ & $26(46)$ & $25(44)$ & $10(48)$ \\
\hline II & $2(9)$ & $11(19)$ & $11(19)$ & $2(9)$ \\
\hline P-value $\left(\chi^{2}\right)$ & \multicolumn{2}{|c|}{0.464} & \multicolumn{2}{|c|}{0.584} \\
\hline \multicolumn{5}{|c|}{ Recurrent disease } \\
\hline Without & $20(95)$ & $41(72)$ & $48(84)$ & $13(62)$ \\
\hline With & $1(5)$ & $16(28)$ & $9(16)$ & $8 \quad(38)$ \\
\hline P-value $\left(\chi^{2}\right)$ & \multicolumn{2}{|c|}{0.027} & \multicolumn{2}{|c|}{0.034} \\
\hline
\end{tabular}

FIGO, International Federation of Gynecology and Obstetrics; EGFR, epidermal growth factor receptor; VEGFR2, vascular endothelial growth factor receptor 2 . 


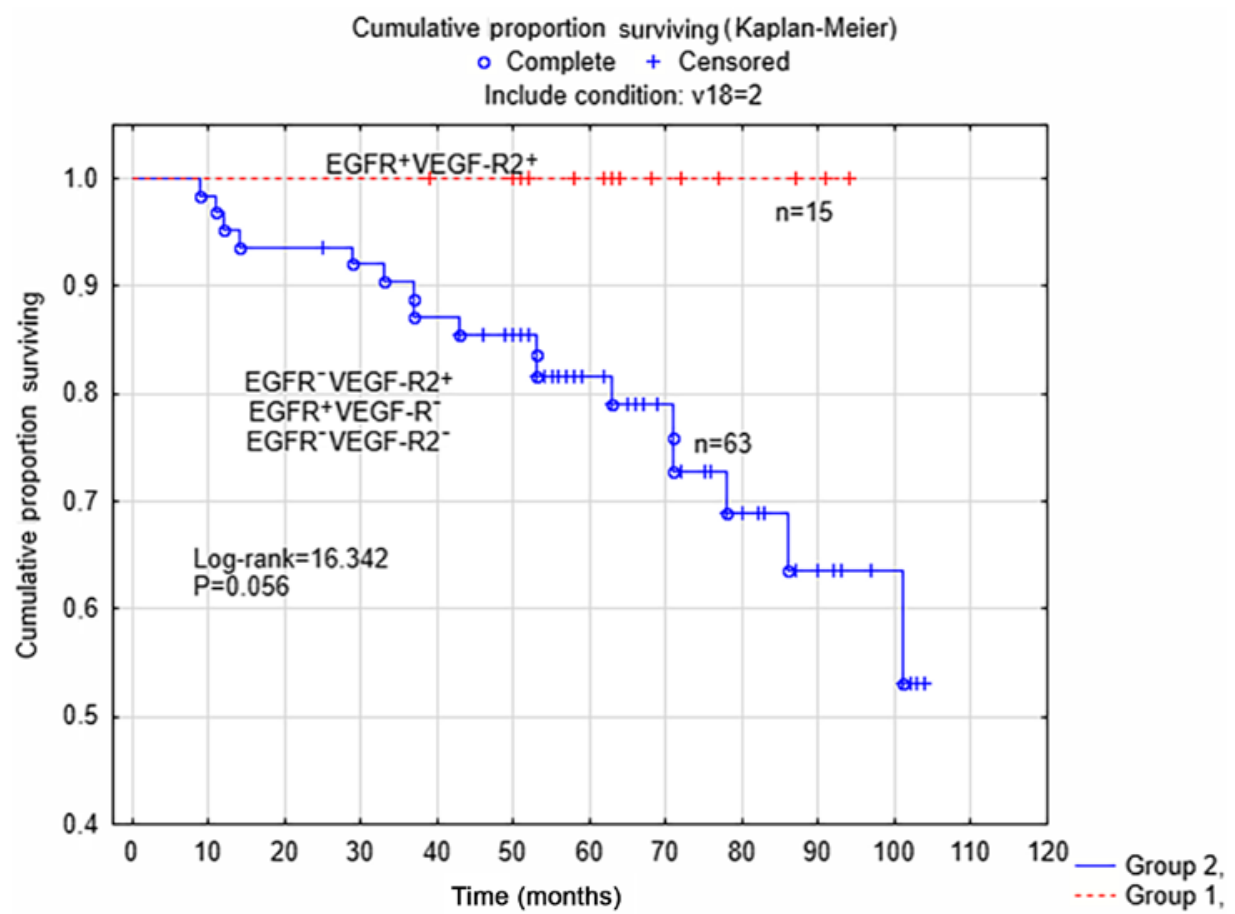

Figure 2. For the subgroup of patients with non-serous tumors $(\mathrm{n}=78)$, there was a trend for improved survival rate in the subgroup of patients ( $\mathrm{n}=15)$ with tumors with concomitant positive expression of EGFR and VEGF-R2, compared with the other three subgroups collectively. EGFR, epithelial growth factor receptor; VEGF-R2, vascular endothelial growth factor receptor 2.

Table V. Cox analysis (univariate and multivariate) with disease-free survival as endpoint ( $\mathrm{n}=130$ patients).

\begin{tabular}{|c|c|c|c|c|c|}
\hline \multirow[b]{2}{*}{ Variable } & \multicolumn{2}{|c|}{ Univariate analysis } & \multicolumn{2}{|c|}{ Multivariate analysis } & \multirow[b]{2}{*}{ P-value } \\
\hline & HR & $95 \% \mathrm{CI}$ & HR & $95 \% \mathrm{CI}$ & \\
\hline Age & 1.016 & $0.986-1.046$ & 1.014 & $0.983-1.046$ & 0.360 \\
\hline Stage (I/II) & 3.318 & $1.655-6.654$ & 3.602 & $1.753-7.403$ & $<0.005$ \\
\hline Type (I/II) & 1.908 & $0.969-3.758$ & 1.799 & $0.899-3.597$ & 0.096 \\
\hline EGFR $^{\mathrm{a}}$ & 0.641 & $0.247-1.663$ & 0.643 & $0.242-1.709$ & 0.376 \\
\hline VEGF-R2 ${ }^{\mathrm{b}}$ & 0.436 & $0.215-0.883$ & 0.322 & $0.153-0.153$ & 0.003 \\
\hline
\end{tabular}

of 21 patients with EGFR-positive tumors, compared with $16(28 \%)$ of the 57 patients with EGFR-negative tumors. Furthermore, the VEGF-R2 status of the non-serous tumors (Table IV) was associated with the type of tumor $(\mathrm{P}=0.016)$ and with recurrent disease $(\mathrm{P}=0.034)$; only nine $(16 \%)$ patients had recurrent disease in the subgroup of 57 patients with VEGF-R2-positive tumors, compared with 8/21 (38\%) patients with VEGF-R2-negative tumors. In the survival analysis limited to patients with non-serous tumors (Fig. 2), there was a trend for improved survival rate $(\mathrm{P}=0.056$; log-rank=16.342) in the subgroup of patients with tumors with concomitant positive expression of EGFR and VEGF-R2 $(n=15)$, compared with the other three subgroups $(n=63)$. There were no differences between the two subgroups of patients according to surgical staging $(\mathrm{P}=0.471)$ or post-surgical treatment $(\mathrm{P}=0.228)$.

Histological subtypes of non-serous tumors. In a separate univariate analysis limited to tumors with endometrioid histology $(n=42)$, it was found that recurrent disease was associated with EGFR negative expression of tumors $(\mathrm{P}=0.023)$. Among the 42 patients, recurrent disease was present in 9/29 (31\%) patients with EGFR-negative tumors, whereas none of the 13 patients with EGFR-positive endometrioid tumors had recurrent disease.

Multivariate analysis. The results for univariate and multivariate Cox analysis with DFS as the endpoint, and logistic regression with recurrent disease as the endpoint, across the entire cohort of patients are, shown in $\mathrm{S} \mathrm{V}$ and VI, respectively. In the first analysis (Table V), FIGO stage and VEGF-R2 status were significant and independent prognostic factors for DFS. With recurrent disease as the endpoint in a multivariate logistic regression analysis, FIGO stage, type of tumor (I/II) and VEGF-R2 status were all independent predictive factors (Table VI).

In the univariate analysis, the results for age and FIGO stage (I/II) listed in Table VI are, also presented in Table V 
Table VI. Predictive factors for recurrent disease via univariate and multivariate logistic regression analysis $(\mathrm{n}=130)$.

\begin{tabular}{|c|c|c|c|c|c|}
\hline \multirow[b]{2}{*}{ Variable } & \multicolumn{2}{|c|}{ Univariate analysis } & \multicolumn{2}{|c|}{ Multivariate analysis } & \multirow[b]{2}{*}{ P-value } \\
\hline & HR & $95 \% \mathrm{CI}$ & HR & $95 \% \mathrm{CI}$ & \\
\hline Age & 1.013 & $0.981-1.047$ & 1.020 & $0.982-1.061$ & 0.289 \\
\hline Stage (I/II) & 7.959 & $2.801-22.617$ & 9.750 & $3.056-31.104$ & $<0.001$ \\
\hline Type (I/II) & 2.456 & $1.099-5.490$ & 2.994 & $1.109-8.804$ & 0.028 \\
\hline $\mathrm{EGFR}^{\mathrm{a}}$ & 0.470 & $0.163-1.358$ & 0.484 & $0.144-1.630$ & 0.237 \\
\hline VEGF-R $2^{b}$ & 0.423 & $0.175-1.018$ & 0.175 & $0.057-0.537$ & 0.002 \\
\hline
\end{tabular}

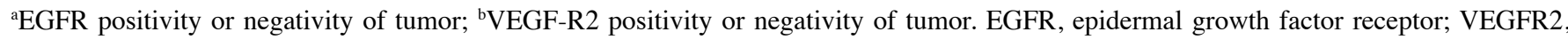
vascular endothelial growth factor receptor 2 .

Table VII. Cox analysis (univariate and multivariate) with disease-free survival as endpoint for patients with non-serous tumors $(n=78)$.

\begin{tabular}{|c|c|c|c|c|c|}
\hline \multirow[b]{2}{*}{ Variable } & \multicolumn{2}{|c|}{ Univariate analysis } & \multicolumn{2}{|c|}{ Multivariate analysis } & \multirow[b]{2}{*}{ P-value } \\
\hline & HR & $95 \% \mathrm{CI}$ & HR & $95 \% \mathrm{CI}$ & \\
\hline Age & 1.016 & $0.977-1.056$ & 1.023 & $0.977-1.072$ & 0.317 \\
\hline Stage (I/II) & 4.315 & $1.654-11.254$ & 4.749 & $1.729-13.041$ & 0.002 \\
\hline Type (I/II) & 2.732 & $1.005-7.427$ & 6.516 & $1.584-26.800$ & 0.009 \\
\hline EGFR $^{\mathrm{a}}$ & 0.205 & $0.027-1.558$ & 0.239 & $0.031-1.840$ & 0.169 \\
\hline VEGF-R2 $2^{b}$ & 0.244 & $0.090-0.662$ & 0.076 & $0.018-0.319$ & 0.319 \\
\hline
\end{tabular}

Table VIII. Predictive factors for recurrent disease (univariate and multivariate logistic regression analysis) for patients with non-serous tumors $(\mathrm{n}=78)$.

\begin{tabular}{|c|c|c|c|c|c|}
\hline \multirow[b]{2}{*}{ Variable } & \multicolumn{2}{|c|}{ Univariate analysis } & \multicolumn{2}{|c|}{ Multivariate analysis } & \multirow[b]{2}{*}{ P-value } \\
\hline & HR & $95 \% \mathrm{CI}$ & HR & $95 \% \mathrm{CI}$ & \\
\hline Age & 1.015 & $0.971-1.060$ & 1.063 & $0.992-1.139$ & 0.075 \\
\hline Stage (I/II) & 10.133 & 2.649-38.759 & 75.965 & $4.212-1369.81$ & 0.003 \\
\hline Type (I/II) & 3.682 & $1.043-12.995$ & 43.836 & 2.088-919.998 & 0.013 \\
\hline $\mathrm{EGFR}^{\mathrm{a}}$ & 0.131 & $0.016-1.096$ & 0.050 & $0.003-0.766$ & 0.028 \\
\hline VEGF-R $2^{\mathrm{b}}$ & 0.298 & $0.094-0.942$ & 0.008 & $0.0002-0.229$ & 0.004 \\
\hline
\end{tabular}

${ }^{a}$ EGFR positivity or negativity of tumor; ${ }^{b}$ VEGF-R2 positivity or negativity of tumor. EGFR, epidermal growth factor receptor; VEGFR2, vascular endothelial growth factor receptor 2 .

of a previous study (10), as the same study population was included in both. For the subgroup of patients with non-serous tumors $(n=78)$, the same analyses were performed and the results are shown in Tables VII and VIII. In the multivariate Cox analysis DFS as the endpoint, only FIGO stage and type (I/II) of tumor were significant and independent prognostic factors for DFS. However, in the logistic regression with recurrent disease as the endpoint (Table VIII), FIGO stage, type (I/II) of tumor, EGFR status and VEGF-R2 status were all significant and independent predictive factors for recurrent disease.

\section{Discussion}

In the present study, the EGFR status alone was associated with tumor grade across the cohort of 131 patients, all in FIGOstage I-II epithelial ovarian cancer, but not with any other clinical or pathological feature or with survival rate. However, recurrent disease was associated with EGFR-negative tumors in the subgroup of patients with non-serous tumors $(n=78)$, and EGFR status was a significant and independent predictive factor for recurrent disease in a multivariate logistic regression analysis for non-serous tumors. The VEGF-R2 status was 
associated with tumor type and recurrent disease; positive staining for VEGF-R2 was, more frequently detected in type II tumors in the entire cohort and in the subgroup of non-serous tumors. The VEGF-R2 status was, according to a multivariate analysis, a prognostic factor for DFS rate in the whole cohort of patients, and also an independent predictive factor for recurrent disease in the whole cohort and the subgroup with non-serous tumors. There were different outcomes in the four subgroups of patients following analysis of the concomitant EGFR and VEGF-R2 status of tumors and in survival analysis; the subgroup of patients $(n=21)$ with tumors exhibiting concomitant positive expression of EGFR and VEGF-R2 had 5-year DFS rates of $100 \%$ across the whole cohort and in survival analysis limited to patients with non-serous tumors $(n=78)$ in the subgroup of patients with tumors with concomitant positive expression of EGFR and VEGF-R2, compared with the other three subgroups. There were no differences between the subgroups of patients in the entire cohort nor those with non-serous tumors, according to staging at primary surgery or post-surgical treatment. Therefore, the different outcomes between the subgroups with respect to EGFR status, VEGF-R2 status and concomitant EGFR and VEGF-R2 status, were most likely explained by their own biological properties.

The EGFR staining was characterized by distinct staining of the cytoplasmic membrane, and positive expression of EGFR was detected in 31/131 (24\%) tumors in the present study. The differences in frequencies of $9-62 \%$ for positive staining of the EGFR protein in previous studies of human ovarian cancer may reflect the use of different antibodies and cutoffs for overexpression (2). In a previous study on ovarian cancer, positive staining for EGFR was detected in 37/106 (34.9\%) patients at FIGO stages I-II, and multivariate analysis revealed the EGFR status of the tumors was an independent and significant prognostic factor (3). The overexpression of EGFR, according to IHC, was present in $39.4 \%$ of the 218 patients with available IHC data in a phase III randomized European Organization for Research and Treatment of Cancer-Gynecological Cancer Group study (16) comparing erlotinib with observations in patients with no evidence of disease progression following first-line platinumbased chemotherapy; the expression of EGFR in tumors was not validated as a poor prognostic marker. It was concluded that, although the EGFR pathway appears to be important in ovarian cancer tumor development, how this pathway may be used for therapeutic benefit remained unclear. By contrast, the results from a meta-analysis (17) of EGFR, including 2,471 patients in 15 studies, showed a significant association between overexpression and poor patient outcome [HR 1.65 (95\% CI 1.25-2.19)]. In a review article entitled Targeting the EGF Receptor for Ovarian Cancer Therapy (18), it was concluded that the overall clinical impact of targeting EGFR and its dimers in ovarian cancer, either with monoclonal antibodies or via inhibition of the tyrosine kinase domain, has been modest in unselected women with advanced or recurrent ovarian cancer. Furthermore, two separate groups have shown an inverse correlation between EGFR and survival rate in ovarian cancer (19).

In another study (20), it was reported that ligand-induced downregulation of EGFR in the $\mathrm{CaOV} 3$ ovarian cancer cell line was possible without tyrosine kinase activity. The downregulation of EGFR without the induction of mitogenic signals, by priming ovarian cancer cells with EGF and EGFR inhibitor PD153035 prior to chemotherapy, was observed in cancer cells that were expected to exhibit increased sensitivity to Taxol-induced cell death. Therefore, it was hypothesized that, by priming with EGFR inhibitors and EGF, certain pathways that lead to cell proliferation and survival can be inhibited by downregulating EGFR. This priming procedure, by sensitizing ovarian cancer cells, was considered to result in improved chemotherapeutic outcome from paclitaxel. This hypothesis may explain the favorable prognostic effect of the positive EGFR status of ovarian tumors on outcomes in the present study, although 105/131 (80\%) of patients received post-surgical paclitaxel. However, no differences in the postsurgical treatments (paclitaxel and carboplatin vs. single drug carboplatin) were found between the two subgroups in the present study. Gavalas et al (4) reported that paclitaxel appeared to have an antiangiogenic effect due to possible increased uptake by endothelial cells in the tumor.

Positive VEGF-R2 staining was observed in 100/130 (77\%) tumors in the present study. This was in line with findings from a study by Nishida et al (6), in which positive staining for VEGF-R2 was detected by IHC in 60/80 (75\%) ovarian tumors from patients at FIGO stages I-IV. However, in this previous study, the high expression of VEGF-R2 in tumors was associated with poorer DFS compared with tumors with negative or low expression of VEGF-R2. In another study (5) of 76 cases of ovarian cancer tumor, a high expression of VEGF-R2 did not have any effect on progression-free or overall survival rates. However, high expression levels of VEGF-R2 were found in $17 / 17(100 \%)$ ovarian tumors at FIGO stages I-II, but only in $39 / 59$ tumors $(66 \%)$ at FIGO stages III-IV. Furthermore, it was reported in a study of 128 patients at FIGO stages I-IV, that patients with high serum levels of VEGF-R2 had improved prognosis, compared with those with low levels of VEGF-R2 (21).

Previous studies $(22,23)$ on various anti-VEGF/VEGF receptor therapies have shown that these agents, when used in combination with chemotherapy, significantly improve survival and response rates in patients. A large number of studies have shown that the inhibition of VEGF or its receptor VEGF-R2 normalizes the tumor vasculature and increases oxygen tension or improves drug penetration. The combination of VEGF-targeted agents with chemotherapy may explain the increased neovascular damage (24). A phenomenon termed 'evasive resistance', which is observed in tumors following anti-VEGF therapy, has been detected, and the suggested mechanisms for the acquisition of this resistance include the induction of angiogenic factors other than VEGF. By using an antibody targeting VEGF-R2 in an animal experiment, it has been shown that vascular regression and tumor reduction occur first, followed by the induction of angiogenesis, leading to tumor regrowth $(25,26)$. These observations may explain why the positive staining of VEGF-R2 in ovarian tumors in the present study had a favorable prognostic effect on survival rates. According to observations from a study on the regulation of angiogenesis (27), it is suggested that vasohibin-1, which acts alone to inhibit multiple different angiogenic factors, may be a more effective inhibitor of angiogenesis than inhibitors which focus on VEGF alone (27).

In a survival analysis of the four subgroups of patients in the present study, with respect to the concomitant EGFR and 
VEGF-R2 status of the tumors, the patients in the subgroup of tumors with concomitant positive expression of EGFR and VEGF-R2 had a DFS rate of $100 \%$ at 5 years. According to the same analysis, the poorest outcome was found for patients belonging to the subgroup of tumors with concomitant negative expression of EGFR and VEGF-R2, with a DFS rate of $64 \%$ at 5 years. However, the main findings from the present study were limited to non-serous tumors and, in further a survival analysis on patients with non-serous tumors $(n=78)$, there was a trend for improved survival rate in the subgroup of patients with concomitant positive expression of EGFR and VEGF-R2, compared with survival rates in the other three subgroups. All 15 patients with non-serous tumors (10/15 patients had endometroid tumors) with concomitant EGFRand VEGF-R2-positive expression had 5-year survival rates of $100 \%$ and were alive 8 years following diagnosis of the primary tumor. A previous study (28) was designed as phase II trial to evaluate the clinical activity and target modulation of vandetanib, designed to inhibit VEGF-R2 and EGFR in women with recurrent and mainly platinum-resistant ovarian cancer. However, $300 \mathrm{mg}$ daily monotherapy with vandetanib had no significant clinical benefit in this disease setting. Proteomic analysis of paired biopsies detected phosphorylated-EGFR and phosphorylated-VEGFR2 in ovarian tumor tissues, but only phosphorylated-EGFR was, measurably inhibited by vandetanib. Apart from targeting the VEGF pathway, novel strategies aim to influence other molecular factors that are involved in tumor angiogenesis (8). In the present study, positive staining for VEGF-R2 in ovarian tumors led to positive results for progression-free survival. Furthermore, in a survival analysis comparing four subgroups following analysis of the status of EGFR and VEGF-R2, the subgroup of patients with concomitant positive expression of EGFR and VEGF-R2 had a 5-year DFS rate of $100 \%$.

In a multivariate logistic regression analysis with recurrent disease as the endpoint, FIGO stage, type (I/II) of tumor and VEGF-R2 status were all independent predictive factors for the entire cohort of patients. In a further multivariate logistic regression analysis with recurrent disease as the endpoint for patients belonging to the subgroup of non-serous tumors $(n=78)$, the FIGO-stage, type (I/II) of tumor, EGFR status and VEGF-R2 status were all significant and independent predictive factors. However, in a multivariate Cox analysis with DFS as the endpoint, in the group of patients with non-serous tumors, only the FIGO-stage and type (I/II) of tumor were significant and independent prognostic factors. The different outcomes of variables between the two forms of multivariate analysis reflect the fact that prognostic factors, but not predictive factors, are dependent of the time interval between diagnosis and analysis.

The limitations of the present study correspond to the relatively limited number of patients included and the method of semi-quantitative analysis used for interpretation, wherein all markers were, dichotomized into negative and positive groups. Preclinical and clinical observations have shown that the process of angiogenesis remains to be fully, elucidated. Therefore, the first concept underlying antiangiogenic therapy was the destruction of tumor vessels; it transpired that, paradoxically, antiangiogenic drugs normalized the vasculature and, as result, offered an improvement in chemotherapeutic delivery. Several trials of anti-angiogenic agents in the front-eline treatment of ovarian cancer have shown positive results for progression-free survival. However, the impact on overall survival rates remains to be fully elucidated. Therefore, one of the challenges in the investigation of ovarian cancer is to identify novel biomarkers for angiogenesis.

\section{Acknowledgements}

Not applicable.

\section{Funding}

No funding was received.

\section{Availability of data and materials}

The datasets used and/or analyzed during the current study are available from the corresponding author on reasonable request.

\section{Authors' contributions}

IS, TS and HA were involved in conceptualization, formal analysis and methodology; they also provided resources, and validation and visualization of the data. IS was involved in data curation and project administration. IS and TS contributed toward the investigation. IS and HÅ supervised the study, and wrote and edited the manuscript.

\section{Ethics approval and consent to participate}

All tissue samples were collected with the patients' informed consent and were in compliance with the Helsinki Declaration(11), and used in accordance with the Swedish Biobank Legislation and Ethical Review Act approved by the Uppsala Ethical Review Board (Uppsala, Sweden; decision ref. UPS-03-477).

\section{Patient consent for publication}

Not applicable.

\section{Competing interests}

The authors declare that they have no competing interests.

\section{References}

1. Siwak DR, Carey M, Hennessy BT, Nguyen CT, McGahren Murray MJ, Nolden L and Mills GB: Targeting the epidermal growth factor receptor in epithelial ovarian cancer: Current knowledge and future challenges. J Oncol 2010: 568938, 2010.

2. Sheng Q and Liu J: The therapeutic potential of targeting the EGFR family in epithelial ovarian cancer. Br J Cancer 104: 1241-1245, 2011.

3. Skirnisdottir I Sorbe B and Seidal T: The growth factor receptors HER-2/neu and EGFR, their relationship, and their effects on the prognosis inearly stage (FIGO I-II) epithelial ovarian carcinoma. Int J Gynecol Cancer 11: 119-129, 2001.

4. Gavalas NG, Liontos M, Trachana SP, Bagratuni T, Arapinis C, Liacos C, Dimopoulos MA and Bamias A: Angiogenesis-related pathways in the pathogenesis of ovarian cancer. Int J Mol Sci 14: 15885-15909, 2013.

5. Klasa-Mazurkiewicz D, Jarząb M, Milczek T, Lipińska B and Emerich J: Clinical significance of VEGFR-2 and VEGFR-3 expression in ovarian cancer patients. Pol J Pathol 62: 31-40, 2011. 
6. Nishida N, Yano H, Komai K, Nishida T, Kamura T and Kojiro M: Vascular endothelial growth factor $\mathrm{C}$ and vascular endothelial growth factor receptor 2 are related closely to the prognosis of patients with ovarian carcinoma. Cancer 101: 1364-1374, 2004.

7. Lassus H, Sihto H, Leminen A, Joensuu H, Isola J, Nupponen NN and Butzow R: Gene amplification, mutation, and protein expression of EGFR and mutations of ERBB2 in serous ovarian carcinoma. J Mol Med (Berl) 84: 671-681, 2006.

8. Maj E, Papiernik D and Wietrzyk J: Antiangiogenic cancer treatment: The great discovery and greater complexity (Review). Int J of Oncol 49: 1773-1784, 2016.

9. Elie C, Geay JF, Morcos M, Le Tourneau A, Girre V, Broët P, Marmey B, Chauvenet L, Audouin J, Pujade-Lauraine E, et al; GINECO Group: Lack of relationship between EGFR-1 immunohistochemical expression and prognosis in a multicentre clinical trial of 93 patients with advanced primary ovarian epithelial cancer (GINECO group). Br J Cancer 91: 470-475, 2004.

10. Skirnisdottir I, Seidal T and Åkerud H: The relationship of the angiogenesis regulators VEGF-A, VEGF-R1 and VEGF-R2 to p53 status and prognostic factors in epithelial ovarian carcinoma in FIGO-stages I-II. Int J Oncol 48: 998-1006, 2016.

11. World Medical Association: WMA Declaration of HelsinkiEthical Principles for Medical Research Involving Human Subjects. https://www.wma.net/policies-post/wma-declarationof-helsinki-ethical-principles-for-medical-research-involvinghuman-subjects.

12. Trimbos JB, Vergote I, Bolis G, Vermorken JB, Mangioni C, Madronal C, Franchi M, Tateo S, Zanetta G, Scarfone G, et al; EORTC-ACTION collaborators. European Organisation for Research and Treatment of Cancer-Adjuvant ChemoTherapy in Ovarian Neoplasm: Impact of adjuvant chemotherapy and surgical staging in early-stage ovarian carcinoma: European Organisation for Research and Treatment of Cancer-Adjuvan ChemoTherapy in Ovarian Neoplasm trial. J Natl Cancer Inst 95: $113-125,2003$.

13. Kononen J, Bubendorf L, Kallioniemi A, Bärlund M, Schraml P, Leighton S, Torhorst J, Mihatsch MJ, Sauter G and Kallioniemi OP: Tissue microarrays for high-throughput molecular profiling of tumor specimens. Nat Med 4: 844-847, 1998.

14. Seidal T, Balaton AJ and Battifora H: Interpretation and quantification of immunostains. Am J Surg Pathol 25: 1204-1207, 2001.

15. Köbel M, Kalloger SE, Boyd N, McKinney S, Mehl E, Palmer C, Leung S, Bowen NJ, Ionescu DN, Rajput A, et al: Ovarian carcinoma subtypes are different diseases: Implications for biomarker studies. PLoS Med 5: e232, 2008.

16. Despierre E, Vergote I, Anderson R, Coens C, Katsaros D, Hirsch FR, Boeckx B, Varella-Garcia M, Ferrero A, Ray-Coquard I, et al; European Organisation for Research and Treatment of Cancer-Gynaecological Cancer Group (EORTCGCG); Groupe d'Investigateurs Nationaux pour les Etudes des Cancers de l'Ovaire (GINECO); Austrian Arbeitsgemeinschaft für Gynäkologische Onkologie (A-AGO); National Cancer Research Institute (NCRI); Australia New Zealand Gynaecological Oncology Group (ANZGOG); Mario Negri Gynecologic Oncology group (MaNGO): Epidermal growth factor receptor (EGFR) pathway biomarkers in the randomized phase III tria of erlotinib versus observation in ovarian cancer patients with no evidence of disease progression after first-line platinum-based chemotherapy. Target Oncol 10: 583-596, 2015.
17. de Graeff P, Crijns APG, Ten Hoor KA, Klip HG, Hollema H, Oien K, Bartlett JM, Wisman GB, de Bock GH, de Vries EG, et al: The ErbB signalling pathway: Protein expression and prognostic value in epithelial ovarian cancer. Br J Cancer 99: 341-349, 2008.

18. Zeineldin R, Muller CY, Stack MS and Hudson LG: Targeting the EGF receptor for ovarian cancer therapy. J Oncol 2010: 414676, 2010.

19. Huang J, Hu W and Sood AK: Prognostic biomarkers in ovarian cancer. Cancer Biomark 8: 231-251, 2010.

20. Cao C, Lu S, Sowa A, Kivlin R, Amaral A, Chu W, Yang H, Di W and Wan Y: Priming with EGFR tyrosine kinase inhibitor and EGF sensitizes ovarian cancer cells to respond to chemotherapeutical drugs. Cancer Lett 266: 249-262, 2008.

21. Komatsu H, Oishi T, Itamochi H, Shimada M, Sato S, Chikumi J, Sato S, Nonaka M, Sawada M, Wakahara M, et al: Serum vascular endothelial growth factor-A as a prognostic biomarker for epithelial ovarian cancer. Int J Gynecol Cancer 27: 1325-1332, 2017.

22. Burger RA: Role of vascular endothelial growth factor inhibitors in the treatment of gynecologic malignancies. J Gynecol Oncol 21: 3-11, 2010.

23. Tang C, Hess K, Jardim DL, Gagliato Dde M, Tsimberidou AM, Falchook G, Fu S, Janku F, Naing A, Piha-Paul S, et al: Synergy between VEGF/VEGFR inhibitors and chemotherapy agents in the phase I clinic.. Clin Cancer Res 20: 5956-5963, 2014.

24. Hicklin DJ and Ellis LM: Role of the vascular endothelial growth factor pathway in tumor growth and angiogenesis (review). J Clin Oncol 23: 1011-1027, 2005.

25. Casanovas O, Hicklin DJ, Bergers G and Hanahan D: Drug resistance by evasion of antiangiogenic targeting of VEGF signaling in late-stage pancreatic islet tumors. Cancer Cell 8: 299-309, 2005.

26. Bergers $G$ and Hanahan D: Modes of resistance to anti-angiogenic therapy. Nat Rev Cancer 8: 592-603, 2008.

27. Takahashi Y, Saga Y, Koyanagi T, Takei Y, Machida S, Taneichi A, Mizukami H, Sato Y, Matsubara S and Fujiwara H: The angiogenesis regulator vasohibin-1 inhibits ovarian cancer growth and peritoneal dissemination and prolongs host survival. Int J Oncol 47: 2057-2063, 2015.

28. Annunziata CM, Walker AJ, Minasian L, Yu M, Kotz H, Wood BJ, Calvo K, Choyke P, Kimm D, Steinberg SM, et al: Vandetanib, designed to inhibit VEGFR2 and EGFR signaling, had no clinical activity as monotherapy for recurrent ovarian cancer and no detectable modulation of VEGFR2. Clin Cancer Res 16: 664-672, 2010. 